\title{
THE
}

1971

\section{Phase Behavior of Models with Competing Interactions}

Jill C. Bonner

University of Rhode Island

J. F. Nagle

Follow this and additional works at: https://digitalcommons.uri.edu/phys_facpubs

Terms of Use

All rights reserved under copyright.

\section{Citation/Publisher Attribution}

Bonner, J. C., \& Nagle, J. F. (1971). Phase behavior of models with competing interactions. J. Appl. Phys. 42(4), 1280-1282. doi: 10.1063/1.1660214

Available at: http://dx.doi.org/10.1063/1.1660214

This Article is brought to you for free and open access by the Physics at DigitalCommons@URI. It has been accepted for inclusion in Physics Faculty Publications by an authorized administrator of DigitalCommons@URI. For more information, please contact digitalcommons-group@uri.edu. 


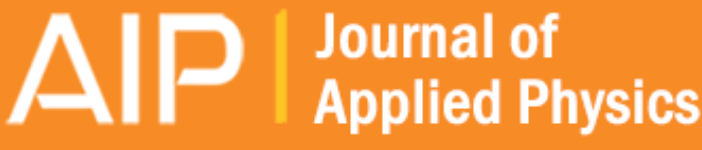

\section{Phase Behavior of Models with Competing Interactions}

J. C. Bonner and J. F. Nagle

Citation: Journal of Applied Physics 42, 1280 (1971); doi: 10.1063/1.1660214

View online: http://dx.doi.org/10.1063/1.1660214

View Table of Contents: http://scitation.aip.org/content/aip/journal/jap/42/4?ver=pdfcov

Published by the AIP Publishing

\section{Articles you may be interested in}

Phase Diagrams of a Potts Model with Competing Binary and Ternary Interactions

AIP Conf. Proc. 1281, 2069 (2010); 10.1063/1.3498356

Phase Diagram Of The Potts Model With Competing Interactions

AIP Conf. Proc. 1150, 407 (2009); 10.1063/1.3192281

On Phase Transitions for p-Adic Potts Model with Competing Interactions on a Cayley Tree

AIP Conf. Proc. 826, 140 (2006); 10.1063/1.2193118

Effect of competing interactions on the critical behavior of $\mathrm{FeCl} 2$

J. Appl. Phys. 84, 386 (1998); 10.1063/1.368040

Solitons and phasons in the Ising model with competing interactions

J. Appl. Phys. 50, 7409 (1979); 10.1063/1.326910

\section{The new SR865 2 MHz Lock-In Amplifier ... \$7950}

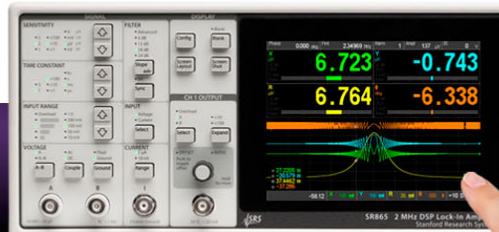
SRS Stanford Research Systems

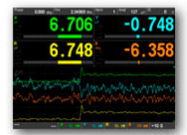

Chart recording

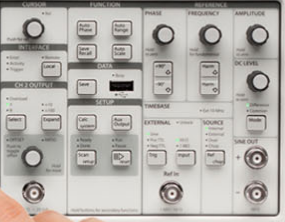

Intuitive front-panel operation

Touchscreen data display

- Save data \& screen shots to USB flash drive

- Embedded web server and iOS app

. Synch multiple SR865s via $10 \mathrm{MHz}$ timebase I/O

-View results on a TV or monitor (HDMl output)

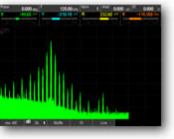

FFT displays

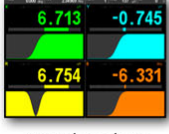

Trend analysis

$1 \mathrm{mHz}$ to $2 \mathrm{MHz}$

$\cdot 2.5 \mathrm{nV} / \sqrt{ } \mathrm{Hz}$ input noise

$.1 \mu$ s to 30 ks time constants

- $1.25 \mathrm{MHz}$ data streaming rate

Sine out with DC offset

GPIB, RS-232, Ethernet \& USB 


\title{
Phase Behavior of Models with Competing Interactions*
}

\author{
J. C. Bonner and J. F. Nagle $\dagger$ \\ Physics Department, Carnegie-Mellon University, Pittsburgh, Pennsylvania 15213
}

\begin{abstract}
Models with competing nearest-neighbor and very long-range interactions are solved cxaclly for several one-dimensional cases, including the usual Ising chain $(I C C I)$, the $X Y$ model $(X Y C I)$, the transverse Ising model $(T I C C I)$, and the spherical model $(S C C I)$. For certain ratios of the competing interaction strengths, $I C C I$ and $X Y C I$ display triple points and two critical points in a field. In addition TICCI has an apparently enclosed phase in an $H-T$ phase diagram; however, this phase is really paramagnetic as can be seen when an extended phase diagram is used. The extended phase diagrams for $I C C I, X Y C I$, and $T I C C I$ display tricritical points and the tricritical exponents have different values from the usual classical values. In contrast to the rich phase behavior of the preceding models, $S C C I$ shows very simple phase behavior, which is directly related to the ground state. Finally, the introduction of a staggered field to $I C C I$ and a simple transformation allows reinterpretation as a metamagnetic model. Using $I C C I$ as a guide the observability of the tricritical exponents is discussed.
\end{abstract}

Interest has recently arisen in two-parameter model Hamiltonians. ${ }^{1,2}$ We have made a theoretical study of a variety of one-dimensional nearest-neighbor models in the presence of a very long-range, equivalent-neighbor (mean field) interaction, and a rich variety of phase behavior has been found when the short-range and long-range interactions compete. Extended phase diagrams plotted in terms of applied field $H$, temperature $T$, and a parameter representing the ratio of longrange to short-range interactions $J_{L R} / J_{S R}$ show an unusual type of critical point called (after Griffiths) a tricritical point, i.e., the meeting point of three second-order critical lines. For our models, the tricritical points display the set of exponents

$$
\begin{array}{cc}
\gamma=\gamma^{\prime}=1, \quad \beta=1 / 4, \quad . \quad \delta=5, \\
\alpha^{\prime}=\frac{1}{2}, & \text { and } \alpha=0,
\end{array}
$$

in contrast to the usual mean-field set.

A feature of interest also is the strong dependence of the resultant phase behavior on the particular type of short-range competing interaction. The Hamiltonian for the linear Ising chain with competing interactions $(I C C I)$ is given as

$$
\begin{array}{r}
\mathfrak{H}_{Y C C I}=2\left|J_{S R}\right| \sum_{i=1}^{N} S_{i}{ }^{z} S_{i+1}{ }^{z}-H \sum_{i=1}^{N} S_{i}{ }^{z}-\left(J_{L R} / N\right) \\
\times \sum_{i=1}^{N} \sum_{j=1}^{N} S_{i}{ }^{z} S_{j}{ }^{2},
\end{array}
$$

and a sketch of the extended phase diagram is shown as Fig. 1. The exact solution of this composite Hamiltonian is quite simple, and the general technique is fairly well known. ${ }^{3}$ Tracing out the phase boundaries in detail, however, may be analytically nontrivial and is most easily done by computer. Special features, however, have been studied analytically.

We observe in Fig. 1 that when the ratio $J_{L R} / J_{S R}$ decreases to a critical value, a triple line appears, resulting from the intersection of two first-order phase surfaces symmetrically situated out in nonzero fields with the
$H=0$ phase surface. At the top of the triple line sits the tricritical point. A qualitatively similar diagram is observed in the case of the linear $X Y$ model $(X Y C I)$ whose Hamiltonian is

$$
\begin{aligned}
\mathfrak{C}_{X Y C Y}=2 J_{S R} & \sum_{i=1}^{N}\left(S_{i}{ }^{x} S_{i+1} x+S_{i}{ }^{y} S_{i+1}{ }^{u}\right) \\
& -H \sum_{i=1}^{N} S_{i}{ }^{z}-\left(J_{L R} / N\right) \sum_{i=1}^{N} \sum_{i=1}^{N} S_{i}{ }^{2} S_{j}{ }^{z},
\end{aligned}
$$

and numerical studies suggest that the linear antiferro-

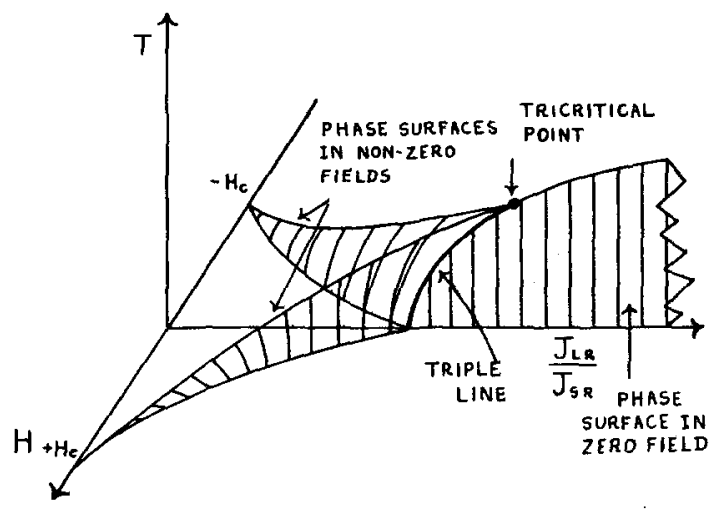

FIG. 1. Extended phase diagram for /CC.

magnetic Heisenberg model also has this type of phase diagram.

However, the Ising model in a transverse field, plus a mean field in the same direction as the applied field, whose Hamiltonian is

$$
\begin{aligned}
& \mathcal{H}_{T I C C I}=2 J_{S R} \sum_{i=1}^{N} S_{i}{ }^{x} S_{i+1}{ }^{x} \\
& -H \sum_{i=1}^{N} S_{i}{ }^{z}-\left(\left|J_{L R}\right| / N\right) \sum_{i=1}^{N} \sum_{i=1}^{N} S_{i}^{z} S_{j}^{z},
\end{aligned}
$$

shows an additional interesting feature, sketched in 
Fig. 2. The triple line "doubles back" on itself, meeting the axis at $T=0$ at a value of $J_{L R} / J_{S R}$ larger than the value corresponding to the tricritical point. Clearly, the shaded area under the triple line indicates a region showing a double phase transition in zero field. ${ }^{4}$ The $B-T$ phase diagram associated with this double phase transition is of further interest, and is shown as Fig. 3. For values of $J_{L R} / J_{S R}$ indicated by the shaded area, an enclosed phase region appears for temperatures below $T_{1}$ at which the system undergoes a first-order transition to a ferromagnetic state. ${ }^{5}$ At first sight this seems surprising, since an enclosed $H-T$ boundary is usually associated with some kind of long-range order, such as antiferromagnetism. Here, however, the enclosed region is paramagnetic, having transverse shortrange order, since in the extended phase diagram corresponding to Fig. 2, it is possible to pass from a hightemperature paramagnetic region to the "enclosed" region without passing through a phase surface.

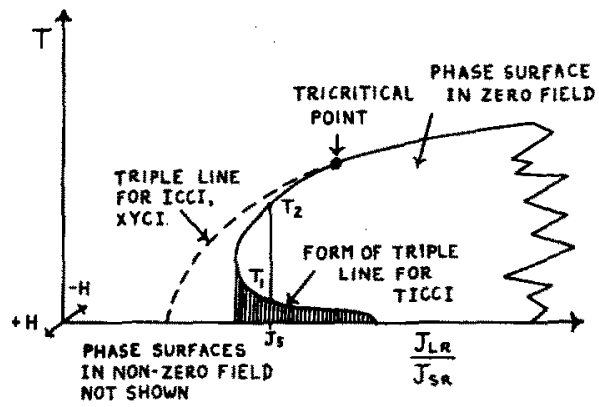

FIG. 2. $H=0$ phase diagram for $T I C C I$. The form of the triple line for $I C C I$ and $X Y C I$ is shown dashed for comparison.

By contrast with the rich variety of phase behavior of the $I C C I, X Y C I$, and $T I C C I$ models, the extended phase diagram for the linear spherical model $(S C C I)$ is very simple. The first-order phase surface in zero field terminates at a value of $J_{L R} / J_{S R}$ such that the ground state changes character from ferromagnetic to antiferromagnetic. There is no tricritical point and no phase surfaces out in a nonzero field. ${ }^{6}$

Of greater experimental interest is further work on $I C C I$ with a staggered applied field in addition to a direct field. ${ }^{7}$ The Hamiltonian (1) then becomes

$$
\begin{aligned}
& \mathfrak{H}_{I C C I S T A G}=2\left|J_{S R}\right| \sum_{i=1}^{N} S_{i}{ }^{2} S_{i+1}{ }^{z}-H \sum_{i=1}^{N} S_{i}{ }^{z} \\
& -H^{\prime} \sum_{i=1}^{N}(-1)^{i} S_{i}{ }^{z}-\left(\left|J_{L R}\right| / N\right) \sum_{i=1}^{N} \sum_{j=1}^{N} S_{i}{ }^{z} S_{j}{ }^{z}
\end{aligned}
$$

Flipping over the spins on one sublattice shows that this model is equivalent to a ferromagnetic nearestneighbor linear chain with a "staggered" type of mean

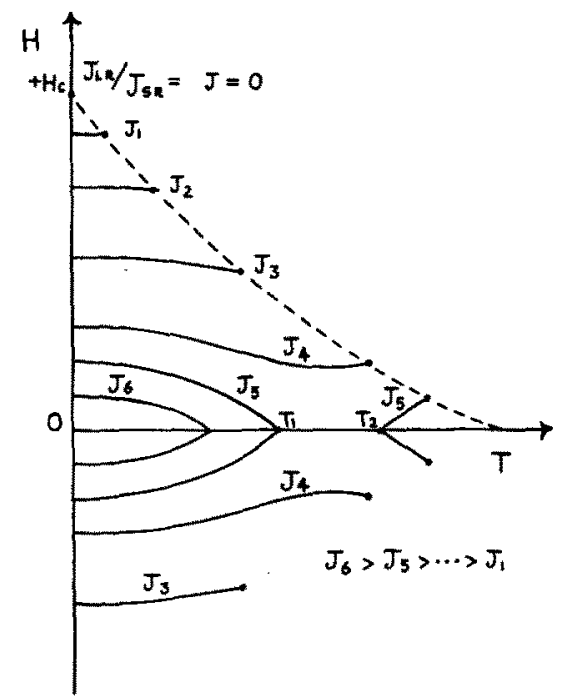

Fig. 3. $H-T$ phase diagram for $T I C C l$ for various values of $J_{L R} / J_{S R}$

field. A simple staggered mean-field model is capable of producing the familiar antiferromagnetic-paramagnetic second-order phase boundary in an $H-T$ plane. The influence of some ferromagnetic short- and long-range interaction causes the low-temperature portion of the boundary to become first order, just as in the case of a classical metamagnet. These features are visible in the extended $H, H^{\prime}, T$ phase diagram shown as Fig. 4 . The first-order portion of the boundary becomes a triple line when an extra degree of freedom, $H^{\prime}$, is considered, since two additional phase surfaces appear for $H \neq 0$. Further, the point at which the boundary changes from first to second order appears as a tricritical point with the same set of tricritical exponents as previously. For

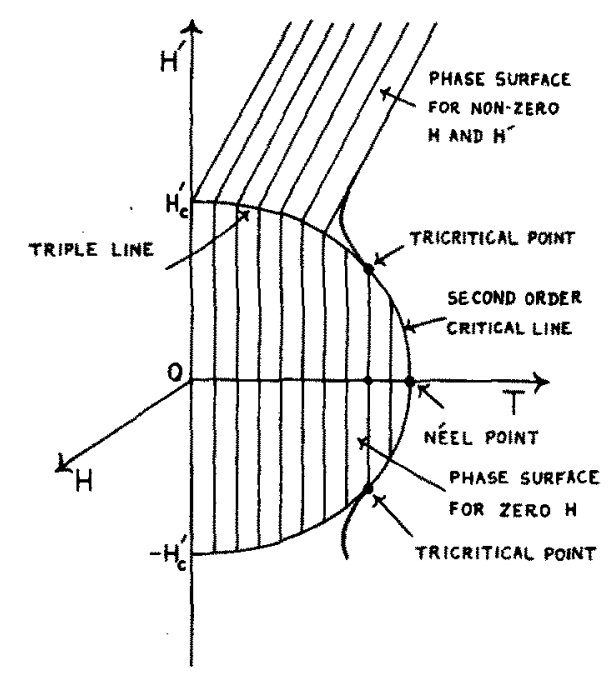

Frg. 4. $H^{\prime}, H, T$ diagram for $I C C I$ in an additional staggered field. 
a particular value of $J_{L R} / J_{S R}$, the two tricritical points for positive and negative $\not^{\prime}$ merge with the Neel point to yield a tetracritical point, i.e., a meeting point of four second-order critical lines. The tetracritical exponents, however, are the same as the tricritical exponents.

Studies on ICCI show that for ordinary critical points near the tricritical point, the apparent exponents for decades of $\log \left|T-T_{c}\right|$ far away from $T_{c}$ look more like tricritical exponents than ordinary mean-field exponents and become mean-field-like only for decades close in to $T_{c}$. This suggests that tricritical exponents may be "dominant" in experimentally accessible regions of temperature near tricritical points and hence tricritical exponents should be experimentally observable.
* Research supported in part by the NSF.

$\uparrow$ Alfred P. Sloan Foundation Fellow.

I P. W. Anderson, G. Yuval, and D. R. Hamann, Phys. Rev. B $1,4464(1970)$. Anderson's theory of the Kondo problem involves long-range $\left(1 / r^{2}\right)$ and nearest-neighbor linear Ising interactions.

2 R. B. Griffiths, Phys. Rev. Lett. 24, 715, 1479 (1970). Griffiths demonstrates the importance of extra interactions for systematizing and predicting the nature of phase transitions.

J. F. Nagle, Phys. Rev. A 2, 2124 (1970).

4 We believe this is the first example of a double-phase transition in one dimension. The double transition "only just" develops for TICCI, and the salient features of Fig. 2 are exaggerated for clarity of presentation.

${ }^{5}$ For the same values of $J_{L R} / J_{S R}$ the system undergoes a further transition at a higher temperature $T_{2}$ to a high-temperature paramagnetic state.

Work performed in collaboration with G. Stell (unpublished). ${ }^{7}$ J. F. Nagle and J. C. Bonner, J. Chem. Phys. (to be published).

\title{
Problem of Using Kink-Point Locus to Determine the Critical Exponent $\beta$
}

\author{
A. ARrotx \\ Depariment of Physics, Simon Fraser University, Burnaby 2, British Columbia
}

\begin{abstract}
It is shown that depending on how one analyzes measurements of the temperature dependence of magnetization near the Curie temperature it is possible to conclude that the critical exponent changes near $T_{c}$. An apparent change in $\boldsymbol{\beta}$ can be obtained from analyzing data generated from an equation of state in which $\beta$ does not change. The mathematical and experimental difficulties in the use of the kink-point locus method are discussed.
\end{abstract}

The "kink-point" method of determining Curie temperatures was used by the Strausborg school during the thirties and apparently originated with Néel. ${ }^{1}$ The basic assumption of the method is that below $T_{\mathrm{c}}$ there is an unsaturated state in which the permeability is very large. In the limit of high permeability any sample with a uniform demagnetizing factor has its magnetization determined by $M=B_{a} / 4 \pi D$, where $B_{a}$ is the applied field. If the high permeability persists until $M=M_{s}(T)$, then for $B_{a}>4 \pi D M_{s}(T)$,

$$
M=\left[M_{s}(T)+\chi B\right] /(1+4 \pi D \chi),
$$

where $\chi$ is the initial susceptibility in the saturated state. For cubic materials where the anisotropy should vanish as a high power of the magnetization on approaching the Curie temperatures, the above model should be close to the experimental situation. One would expect that for measurements in constant applied field $M$ would remain constant with increasing temperature until $T=T_{B}$, where $T_{B}$ is the temperature at which $M_{s}\left(T_{B}\right)=B_{a} / 4 \pi D$. Above $T_{B}, d M / d T$ should have a finite value except in the limit as $T \rightarrow T_{c}$. Thus there should be a break point which should allow the determination of $T_{B}$ for each $B_{a}$; hence one should be able to obtain $M_{s}(T)$ in this way. Alternatively, one

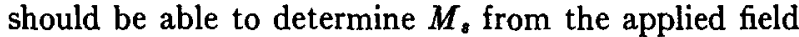
dependence of $4 \pi D M / B_{a}$ at constant temperature; $4 \pi D M / B_{a}$ should remain constant in the unsaturated region and then start to decrease with a finite slope for $B_{a}>4 \pi D M_{s}(T)$. Both methods suffer in practice from two effects. Very near $T_{c}$ the vanishing initial derivatives and the high curvatures make it very difficult to obtain $M_{s}(T)$ with adequate precision. Further from $T_{c}$, where the derivatives are finite and slowly varying, the fundamental assumption of the method, namely, very high permeability for $M<M_{s}(T)$, is no longer sufficiently true. That the permeability is limited by anisotropy, possibly arising from defects, is indicated by the observation that the decrease in permeability in the unsaturated region is more noticeable in a polycrystalline $\mathrm{Ni}$ sample than in a single-crystal Ni sample. ${ }^{2}$ The effects of these things on the temperature dependence of the magnetization ${ }^{3}$ of a nickel spherical single crystal in constant applied fields are shown in Fig. 1. For $240 \mathrm{G}$ the anisotropy effect shows a decrease in $M$ some $0.2 \mathrm{deg}$ below what I consider to be $T_{B}$. For $18 \mathrm{G}$, there is an intrinsic curvature in addition to that arising from anisotropy (much smaller at $18 \mathrm{G}$ than at $240 \mathrm{G}$ ). This is because $\chi$ depends quite markedly on temperature and field near $T_{c}$. At $240 \mathrm{G}$ one can use a linear 